\title{
EFICÁCIA DO CURATIVO HIDROCOLÓIDE EM RELAÇÃO AO FILME TRANSPARENTE NA PREVENÇÃO DE LESÕES POR PRESSÃO
}

Tatyanne Silva Rodrigues¹, Camila Aparecida Pinheiro Landim Almeida², Pétterson Danilo de Oliveira Lima Goiano ${ }^{3}$, Valéria Maria Silva Nepomuceno', Carliane da Conceição Machado Sousa', Mariana Lustosa de Carvalho ${ }^{1}$

Objetivo: Identificar as evidências científicas disponíveis sobre a eficácia do curativo hidrocolóide em relação ao filme transparente na prevenção de lesões por pressão. Metodologia: Revisão integrativa da literatura, realizada em abril de 2017, em estudos primários, publicados entre 2007 a 2017, nos idiomas português, espanhol e inglês. A busca dos estudos foi realizada nas bases de dados SciELO, BDENF, LILACS, MEDLINE, PubMed, Web of Science e CINAHL, direcionada pela questão de pesquisa: Qual a eficácia do curativo hidrocolóide em relação ao filme transparente na prevenção de lesões por pressão? Resultados: Foram encontrados 780 artigos científicos, que após avaliação quanto à adequação aos critérios de inclusão, duplicidade e conteúdo, obteve-se uma amostra final de seis artigos. Conclusões: As evidências da maioria dos estudos apontaram a superioridade do filme transparente em relação ao hidrocolóide na prevenção de lesões, por ser mais eficiente.

Descritores: Úlcera por pressão, Prevenção, Bandagens

\section{EFFECTIVENESS OF THE HYDROCOLLOID DRESSING COMPARED TO THE TRANSPARENT FILM IN THE PREVENTION OF PRESSURE LESIONS}

Objective: To identify the available scientific evidence on the effectiveness of the hydrocolloid dressing in relation to the transparent film in the prevention of pressure lesions. Methodology: Integrative review of the literature, conducted in April 2017, in primary studies, published between 2007 and 2017, in Portuguese, Spanish and English. Results: A total of 780 scientific articles were found, which, after evaluating the adequacy of the criteria for inclusion, duplicity and contemplation of the proposed theme, obtained a final sample of 6 articles. The results of most studies have pointed to the superiority of the transparent film in relation to the hydrocolloid in the prevention of lesions, because it is more efficient and effective. Conclusions: Although we can estimate the efficacy of the transparent film on the hydrocolloid, we did not find studies with applicability at home.

Descriptors: Pressure ulcer, Prevention, Bandages

\section{EFECTIVIDAD DEL CURATIVO HIDROCOLÓIDE VERSUS PELÍCULAS TRANSPARENTES EN LA PREVENCIÓN DE LESIONES POR PRESIÓN}

Objetivo: Identificar las evidencias científicas disponibles sobre la efectividad del curativo hidrocoloide versus película transparente en la prevención de lesiones por presión. Metodología: Revisión integrativa de la literatura, realizada en abril de 2017, en estudios primarios, publicados entre 2007 a 2017, en los idiomas portugués, español e inglés. Resultados: Se encontraron 780 artículos científicos, que tras evaluación en cuanto a la adecuación a los criterios de inclusión, duplicidad y contemplación al tema propuesto, se obtuvo una muestra final de 6 artículos. Los resultados de la mayoría de los estudios apuntaron la superioridad de la película transparente en relación al hidrocoloide en la prevención de lesiones, por ser más efectivo y económico. Conclusiones: A pesar de poder estimar la efectividad de la película transparente sobre el hidrocoloide, serecomienda realizar estudios adicionales, para reafirmar la eficacia de las coberturas, ya que tampoco se encontraron estudios con aplicabilidad en el ámbito domiciliar.

Descriptores: Úlcera por presión, prevención, vendajes.

Descriptores: Úlcera por pressão, Prevenção, Bandagens 
As lesões por pressão (LPP) representam um grave problema de saúde, por elevarem as taxas de internação, colaborando para o crescimento da morbimortalidade e tratamentos com altos custos para os serviços de saúde(1). São definidas pelo National Pressure Ulcer Advisory Panel (NPUAP) como um dano na pele e/ou tecidos moles subjacentes, comumente localizadas sobre uma proeminência óssea ou relacionada a um dispositivo médico ou outro objeto, resultantes na maioria das vezes, por pressão, fricção, cisalhamento e fatores intrínsecos do paciente, instando-se de forma gradual, por meio de um processo de isquemia tecidual, que resulta em uma lesão de pele, seja em pele íntegra ou em úlcera aberta, com várias classificações, dependendo do tipo de tecido acometido e aspecto da lesão(2).

Em abril de 2016, nos Estados Unidos, o órgão americano NPUAP alterou o termo úlcera por pressão para lesão por pressão, bem como, os estágios do sistema de classificação de algarismos romanos para algarismos arábicos e adição de novas definições ${ }^{(3)}$. No que se refere as alterações no sistema de classificação das LPP, estas foram distribuidas da seguinte maneira: Estágio 1; Estágio 2; Estágio 3; Estágio 4; Lesão não classificável; Lesão tissular profunda; e Lesão relacionada a dispositivos médicos e em membranas mucosas $^{(4)}$

Dentreosfatoresassociadosaoriscodedesenvolvimento de LPP, destacam-se a hipertensão arterial sistêmica; diabetes; inconsciência; imobilização; insuficiência sensorial; perda de função motora; incontinência urinária e/ ou fecal; deficiências nutricionais; índice de massa corporal alterados; doenças circulatórias; imunodeficiência; uso de corticoides por tempo prolongado; tabagismo; e alterações do nivel de consciência ${ }^{(5)}$.

A ocorrência de LPPé considerada um indicador negativo da qualidade da assistência prestada, pois apesar dos fatores extrínsecos não serem os únicos causadores das lesões, estes quando associados aos fatores intrínsecos, apresentam grande influência para a ocorrência da lesão(6). Assim, para facilitar a investigação dos profissionais de saúde frente aos fatores comumente associados ao desenvolvimento das LPP e estratificar seu risco, existem escalas de avaliação de risco, eficazes tanto no âmbito hospitalar, como em domicílio(2). Após os pacientes serem avaliados quanto ao risco de LPP, medidas preventivas devem ser tomadas, e dentre estas, o uso de coberturas protetoras como o filme transparente e hidrocolóide são recomendadas e podem apresentar bons resultados ${ }^{(7)}$

Dessa forma, considerando que as lesões por pressão retratam a qualidade dos cuidados de enfermagem prestados, faz-se necessário que os enfermeiros trabalhem exaustivamente esta problemática, tornando-a um tema de grande relevância para a sua prática clínica e sustentadas nas melhores evidências ${ }^{(8)}$. Assim, o presente estudo tem o objetivo de Identificar as evidências científicas disponiveis sobre a eficácia do curativo hidrocolóide em relação ao filme transparente na prevenção de lesões por pressão.

\section{METODOLOGIA}

Revisão integrativa da literatura, a qual permitiu sintetizar estudos com diferentes métodos de pesquisa, para a obtenção de conclusões de um tema específico e incorporação das evidências identificadas na prática clínica. Para a operacionalização dessa revisão, utilizaram-se as seguintes etapas: identificação do tema e seleção questão de pesquisa; estabelecimento de critérios para inclusão/ exclusão dos estudos; definição das informações a serem extraídas; avaliação dos estudos incluídos, interpretação dos resultados e apresentação da revisão final ${ }^{(9)}$.

Para elaboração da questão de pesquisa, utilizou-se a estratégia $\mathrm{PICO}{ }^{(10)}$, em que P refere-se ao problema (Lesões por pressão); I refere-se à intervenção (Hidrocolóide); C à comparação (Hidrocolóide x Filme transparente); e O caracteriza o resultado esperado (Prevenção das lesões), compondo assim, a seguinte questão: Qual a eficácia do curativo hidrocolóide em relação ao filme transparente na prevenção de lesões por pressão?

Os descritores selecionados foram: úlcera por pressão (pressure ulcer; úlcera por presión); prevenção (prevention; prevención); bandagens (bandages; vendajes). Vale ressaltar que apesar de ter tido mudança do termo úlcera por pressão para lesão por pressão, este termo ainda não faz parte dos Descritores em Ciências da Saúde (DecS). Entretanto, os demais descritores são DeCs e Medical Subject Headings (MeSH), conforme consulta realizada, os quais foram associados utilizando o operador booleano and

O levantamento bibliográfico foi realizado na Scientific Electronic Library Online (SciELO), Banco de Dados em Enfermagem (BDENF), Literatura Latino-Americana e do Caribe em Ciências da Saúde (LILACS), Medical Literature Analysis and Retrieval System Online (MEDLINE), United States National Library of Medicine National Institutes of Health (PubMed), Web of Science e Cumulative Index to Nursing and Allied Health Literature (CINAHL), tendo como critérios de inclusão dos estudos: artigos primários em texto completo gratuito, disponíveis nos idiomas português, inglês e espanhol, publicados entre os anos de 2007 a 2017. A justificativa do critério de seleção de artigos, se deu pelo fato de que estudos que analisem o uso do hidrocolóide e filme transparente para prevenção de lesões, se intensificaram nos últimos 10 anos $^{(11)}$. 
A busca dos artigos científicos foi realizada no mês de abril de 2017, obtendo-se um total de 780 artigos científicos. Deste quantitativo, os artigos foram avaliados quanto a qualidade e adequação aos critérios de inclusão, duplicidade entre as bases, excluindo-se 645 artigos. Posteriormente, foi realizada a leitura dos títulos e resumos, a fim de averiguar a contemplação ao tema, enquadrandose assim, seis artigos para a análise final, conforme mostra a figura 1.

Figura 1 - Fluxograma de identificação, seleção e inclusão dos artigos, para o desenvolvimento da pesquisa. Teresina, Piauí, Brasil, 2017.

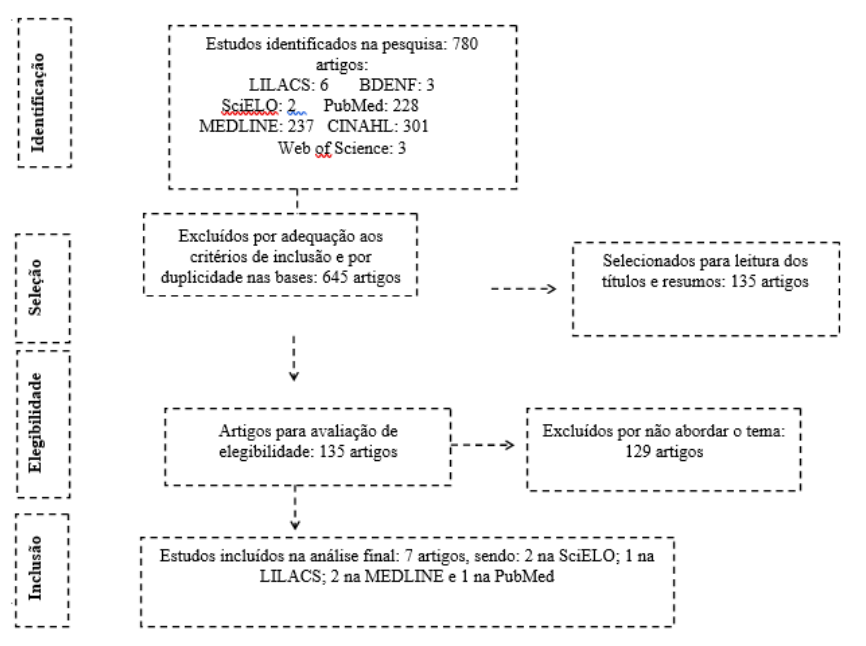

Fonte: Biblioteca Virtual em Saúde, SciELO, PubMed, Web of Science e CINAHL

A extração dos dados dos estudos selecionados será executada por meio da utilização de um instrumento de coleta de dados adaptado, proposto e validado por pesquisadoras da Enfermagem ${ }^{(12)}$. Tal instrumento contempla a identificação do artigo, ano, local do estudo, características/avaliação do rigor metodológico e nível de evidência.

\section{RESULTADOS}

Dos seis estudos selecionados, três (50\%) foram publicados no ano de 2015; dois (33,3\%) em 2016 e um $(16,7)$ em 2013. Diante deste quantitativo, percebe-se que apesar da alta prevalência de lesões por pressão, as publicações não acompanham essa evolução, em especial, as que avaliam a aplicabilidade/efetividade de coberturas protetoras.

Outro fator que pode estar relacionado é o fato de que os estudos relacionados a prevenção de LPP, buscam de forma mais ampla, identificar os cuidados realizados para a prevenção, e quando se busca avaliar a efetividade de curativos, por existirem vários tipos de coberturas e os artigos que avaliam tal intervenção serem em maior número internacionais, pouco se aborda sobre as coberturas em questão neste estudo, já que são mais utilizadas no Brasil(13).

No que se refere ao delineamento dos estudos, verifica-se que os artigos são provenientes de ensaios clínicos controlados, estudos analíticos e transversais, respectivamente, os quais tratavam em sua maioria, sobre a efetividade do filme transparente e hidrocolóide para a prevenção de LPP, com ênfase no número de lesões desenvolvidas após a aplicação das coberturas e na avaliação do custo-efetividade.

Diante da avaliação da efetividade do filme transparente e hidrocolóide na prevenção de LPP, pode-se verificar que o filme transparente foi mais efetivo. $O$ hidrocolóide apresentou-se efetivo em um estudo, entretanto, vale ressaltar, tal cobertura foi avaliada de forma isolada no mesmo. E, em outro estudo, a maior efetividade foi dada a cobertura de ceramida quando comparada ao hidrocolóide. Para a análise e sintese dos artigos selecionadas, utilizouse um quadro sinóptico, construido para esse fim (Quadro 2).

Quadro 1 - Síntese dos estudos primários incluídos na revisão integrativa. Teresina, Piauí, Brasil, 2017.

\begin{tabular}{|c|c|c|c|c|c|}
\hline $\begin{array}{l}\text { Titulo do } \\
\text { artigo }\end{array}$ & Autores/Ano & $\begin{array}{l}\text { Delineamentc } \\
\text { do estudo }\end{array}$ & $\begin{array}{l}\text { Amostra } \\
\text { analisada }\end{array}$ & $\begin{array}{l}\text { Curativo } \\
\text { utilizado }\end{array}$ & $\begin{array}{l}\text { Resultados/ } \\
\text { Conclusöes }\end{array}$ \\
\hline $\begin{array}{l}\text { Custos de } \\
\text { coberturas para } \\
\text { a prevenção } \\
\text { de úlcera por } \\
\text { pressāo sacral }\end{array}$ & $\begin{array}{l}\text { INOUE: } \\
\text { MATSUDA, } 2016\end{array}$ & Estudo analitico & 25 pacientes & $\begin{array}{l}\text { Filme } \\
\text { transparente e } \\
\text { hidrocolóide }\end{array}$ & $\begin{array}{l}\text { O filme } \\
\text { transparente } \\
\text { demostrou-se } \\
\text { uma op̧ão } \\
\text { economica } \\
\text { e eficaz para } \\
\text { prevenir lesões } \\
\text { por pressão } \\
\text { sacrais em } \\
\text { pacientes } \\
\text { criticos e que o } \\
\text { motivo que levou } \\
\text { as trocas foi o } \\
\text { deslocamento. }\end{array}$ \\
\hline $\begin{array}{l}\text { Managing } \\
\text { pressure ulcers } \\
\text { and moisture } \\
\text { lesions with new } \\
\text { hydrocolloid } \\
\text { technology }\end{array}$ & $\begin{array}{l}\text { LINTHWAITE; } \\
\text { BETHELL, } 2016\end{array}$ & Estudo analitico & 10 pacientes & Hidrocolóide & $\begin{array}{l}\text { O hidrocolóide } \\
\text { mostrou- } \\
\text { se efetivo, } \\
\text { reduzindo os } \\
\text { tempos de } \\
\text { cicatrização, } \\
\text { os gastos com } \\
\text { tratamentos } \\
\text { adicionais e } \\
\text { facilitando a } \\
\text { regular inspeção } \\
\text { das áreas } \\
\text { lesionadas. }\end{array}$ \\
\hline $\begin{array}{l}\text { Avaliação } \\
\text { de custo- } \\
\text { efetividade de } \\
\text { dois tipos de } \\
\text { curativos para } \\
\text { prevenção } \\
\text { de úlcera por } \\
\text { pressão }\end{array}$ & $\begin{array}{l}\text { INOUE: } \\
\text { MATSUDA. } 2015\end{array}$ & Estudo analitico & 25 pacientes & $\begin{array}{l}\text { Filme } \\
\text { transparente e } \\
\text { hidrocolóide }\end{array}$ & $\begin{array}{l}\text { O custo- } \\
\text { efetividade } \\
\text { do filme } \\
\text { transparente } \\
\text { foi maior do que } \\
\text { o hidrocolóide } \\
\text { na prevenção } \\
\text { de lesão sacral, } \\
\text { com diferença } \\
\text { de mais de } 100 \% \\
\text { do preço no } \\
\text { desfecho final. }\end{array}$ \\
\hline
\end{tabular}




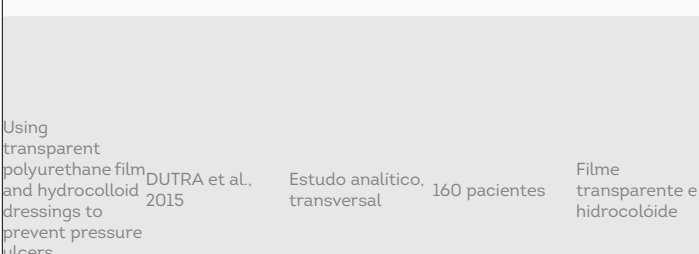

Os resultados

indicam

que o filme

apresentou

melhor

desempenho

eficácia do que

o curativo de

hidrocolóide na

prevenção do

envolvimento pressão.

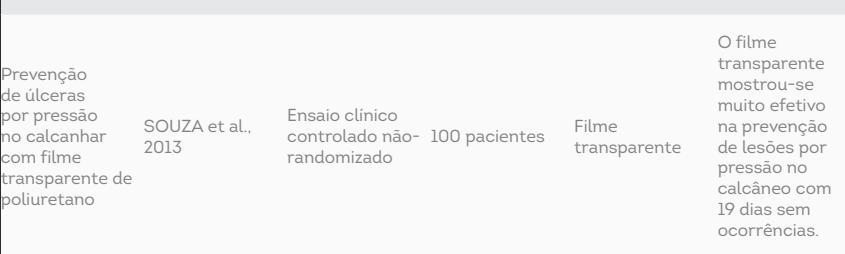

Fonte: Biblioteca Virtual em Saúde, SciELO, PubMed, Web of Science e CINAHL

\section{DISCUSSÃO}

As LPP são consideradas uma grave alteração da integridade da pele e como um indicador de qualidade dos cuidados de enfermagem prestados, já que são esses profissionais, que prestam uma assistência direta aos pacientes cotidianamente ${ }^{(14)}$. São comumente observadas em vários países do mundo, sobretudo, em pacientes idosos que apresentam restrição de mobilidade, e avaliado como uma das mais caras intervenções médico-cirúrgicas aplicadas nesse público ${ }^{(15)}$. A maior prevalência de idosos desenvolverem LPPs, se dá pela menor produção de colágeno, redução da elasticidade, má vascularização(13).

Para a prevenção de LPP em pacientes susceptiveis, é necessário a aplicação de coberturas protetoras em áreas de maior pressão e de proeminências ósseas ${ }^{(14)}$. Dentre as abordagens realizadas para evitar tais lesões, vem sendo utilizado diferentes tipos de curativos, que promovem a proteção da pele e redução das forças de fricção e cisalhamento sobre a pele $e^{(15)}$

Existem vários tipos de curativos disponíveis no mercado para prevenção de LPP, dentre eles, o filme transparente e o hidrocolóide, são os mais utilizados no Brasil e estão disponíveis para uso desde o início da década de $80^{(13)}$. Dessa forma, sob essa constatação, há cada vez mais, a necessidade de ampliar os recursos opcionais para a prevenção de LPP, a fim de reduzir as complicações, desconfortos e altos custos com tratamentos ${ }^{(16)}$.

Assim, considerando há necessidade de racionalização dos recursos financeiros nas instituições de saúde e que as cobertura são indispensáveis para a prevenção de LPPs, torna-se mais lucrativo financeiramente o uso de filme transparente, que se apresentou no estudo realizado na Unidade de Terapia Intensiva (UTI), uma economia média de $\mathrm{R} \$ 167,00$ por paciente, em relação a outras coberturas analisadas ${ }^{(14)}$.

Estudo realizado no Paraná, na Região Sul do Brasil(17) identificou que o filme transparente foi 5,5 vezes mais econômico, quando comparado com a placa hidrocolóide, logo que ofereceu menor custo de aquisição associado a maior quantidade de pacientes assistidos, tendo uma relação de custo-efetividade a cada lesão evitada de cerca de $R$ \$29,00 e 272,00 reais, para o filme e hidrocolóide, simultaneamente.

Ensaio clinico controlado com 100 pacientes de um hospital universitário brasileiro que faziam uso de filme transparente para prevenir lesões por pressão em calcâneos(11), observou-se que a incidência de lesões foi de $6 \%$ na intervenção experimental e $18 \%$ na intervenção controle, sendo possivel afirmar que, embora o paciente possa desenvolver lesões, a possibilidade de ocorrência foi significantemente mais elevada naqueles que não fazem o uso do filme transparente.

Dado que corrobora com o estudo realizado na UTI de Passos em 2015(13), que os resultados indicam que o filme transparente apresentou melhor desempenho e teve maior eficácia do que o curativo de hidrocolóide na prevenção de LPP. No entanto, apesar dessa alta efetividade do filme transparente, em relação ao hidrocolóide, em um estudo de caso realizado em 2016(17), mostrou que o hidrocolóide foi efetivo na prevenção de LPP, agindo na redução do tempo de cicatrização, nos custos com tratamentos adicionais e permitindo a frequente inspeção das áreas lesionadas. Todavia, vale ressaltar, que essa superioridade do hidrocolóide, pode ser justificada por ter sido avaliada isoladamente neste estudo.

Já na pesquisa realizada com 97 pacientes internados em um hospital chinês(15), que avaliou o hidrocolóide com o curativo com ceramida, a segunda opção foi mais eficaz na prevenção de lesões do que a primeira, o que tornase importante, que esse tipo de cobertura seja avaliado em comparação ao filme transparente, pois no presente estudo, mostrou-se a intervenção mais custo-efetiva.

Nesse cenário, o enfermeiro, como líder da equipe de enfermagem, precisa aprimorar suas habilidades de gerenciamento dos serviços e da assistência, com foco na aquisição e melhoria dos recursos físicos, tecnológicos 
e humanos, para a maior segurança dos paciente e profissionais envolvidos nesse processo, e no intuito de prevenir as LPPs, o uso de coberturas protetoras, torna-se uma tecnologia de trabalho inovadora e eficaz ${ }^{(14)}$.

O presente estudo evidencia que a melhor cobertura a ser utilizada na prevenção de LPP é o filme transparente. Esse tipo de discussão ainda é considerada incipiente, frente os altos indices de morbimortalidade ocasionados por essas lesões.

Dessa forma, o estudo teve como limitações a escassez de produções que respondessem à questão de pesquisa, como também não serem identificados estudos que retratassem a aplicabilidade de ambas coberturas analisadas no âmbito domiciliar. O que tornou necessária, a realização de estudos adicionais, em especial, os que avaliam a eficácia de coberturas protetoras. Outra limitação é que não foram consideradas as capacitações dos profissionais de saúde para o manuseio e manutenção de tais coberturas.

\section{CONCLUSÕES}

As evidências da maioria dos estudos apontaram a superioridade do filme transparente em relação ao hidrocolóide na prevenção de lesões. Ressalta-se que também é mais eficiente por apresentar menores custos de aquisição e manutenção. Ressalta-se ainda, a necessidade de avaliação da eficácia do filme transparente, em comparação com outras coberturas protetoras disponíveis no mercado, tanto no âmbito hospitalar e domiciliar. Sugere-se assim, ampliar as orientações acerca dessa temática nas instituições de saúde e em domicílio, criando mecanismos eficazes de comunicação entre profissionais de saúde e os cuidadores, para que assim, seja garantida a continuidade do cuidado e a segurança do paciente.

\section{REFERÊNCIAS}

1- Pott FS, Ribas JD, Silva OBMda, Souza TS, Danski MTR, Marineli MJ. Algoritmo de prevenção e tratamento de Úlcera Por Pressão. Cogitare Enferm [Internet]. 2013 [cited 2017 Jul 20]; 18(2):238-244. Available from: https://revistas.ufpr.br/cogitare/ article/view/26085

2- Borghardt AT, Prado TN, Bicudo SDS, Castro DS, Bringuente MEO. Pressure ulcers in critically ill patients: incidence and associated factors. Rev Bras Enferm [Internet]. 2016 [cited 2017 Jul 20]; 69(3):460-467. Available from: http://www.scielo.br/scielo. php?script=sci_arttextEpid=S0034-716720160003004608lng=en. http://dx.doi. org/10.1590/0034-7167.2016690307i.

3- Moraes JT. Conceito e Classificação de Lesão por Pressão: Atualização do National Pressure Ulcer Advisory Panel. Enferm Cent O Min [Internet]. 2016 [cited 2017 Jul 20] 6(2):2292-2306. Available from: http://www.seer.ufsj.edu.br/index.php/recom/article/ view/1423

4- Olkoski E, Assis GM. Aplicação de medidas de prevenção para úlceras por pressão pela equipe de enfermagem antes e após uma campanha educativa. Esc Anna Nery [Internet]. 2016 [cited 2017 Jul 20]; 20(2):363-369. Available from: http://www.scielo. br/scielo.php?script=sci_arttext\&pid=S1414-81452016000200363\&lng=en. http:// dx.doi.org/10.5935/1414-8145.20160050.

5- Moraes GLA. Aplicação de protocolo de prevenção de úlcera por pressão no contexto domiciliar: uma trajetória percorrida. Cogitare Enferm [Internet]. 2013 [cited 2017 Jul 20]; 18(2):387-391. Available from: https://revistas.ufpr.br/cogitare/article/ view/32590

6- Lima AFC, Castilho V. Mobilização corporal para prevenção de úlceras por pressão: custo direto com pessoal. Rev Bras Enferm [Internet]. 2015 [cited 2017 Jul 20]; 68(5):930-936. Available from: http://www.scielo.br/scielo.php?script=sci_ arttext\&pid=S0034-71672015000500930\&lng=en. http://dx.doi.org/10.1590/0034$7167.2015680523 i$

7- Moro JV. Caliri MHL. Úlcera por pressão após a alta hospitalar e o cuidado em domicilio. Esc Anna Nery [Internet]. 2016 [cited 2017 Jul 20]: 20(3):e20160058. Available from: http://www.scielo.br/pdf/ean/v20n3/1414-8145-ean-20-03-20160058.pdf

8- Freitas JPC. Alberti LR. Aplicação da Escala de Braden em domicilio: incidência e fatores associados a úlcera por pressão. Acta paul enferm [Internet] 2013 [cited 2017 Jul 20]; 26(6):515-521. Available from: http://www.scielo.br/scielo.php?script=sci arttext\&pid=S0103-21002013000600002\&lng=en. http://dx.doi.org/10.1590/S010321002013000600002.

9- Mendes KDS, Silveira RCCP, Galvão CM. Revisão integrativa: método de pesquisa para a incorporação de evidências na saúde e na enfermagem. Texto contexto enferm [Internet]. 2008 [cited 2017 Jul 20]; 17(4):758-764. Available from: http://www.scielo. br/scielo.php?script=sci_arttext\&pid=S0104-07072008000400018\&lng=en. http:// dx.doi.org/10.1590/S0104-07072008000400018.
10- Santos CMC, Pimenta CAM, Nobre MRC. A estratégia PICO para a construção da pergunta de pesquisa e busca de evidências. Rev Latino-Am Enfermagem. [Internet]. 2007 [cited 2017 Jul 20]: 15(3):508-511. Available from: http://www.scielo.br/scielo. php?script=sci_arttext\&pid=S0104-11692007000300023\&lng=en. http://dx.doi. org/10.1590/S0104-11692007000300023.

11- Souza TS, Danski MTR, Johann DA, Lazzari LSM, Mingorance P. Prevenção de úlceras por pressão no calcanhar com filme transparente de poliuretano. Acta paul enferm. [Internet]. 2013 [cited 2017 Jul 20]: 26(4):345-352. Available from: http://www. scielo.br/scielo.php?script=sci_arttext\&pid=\$0103-21002013000400008\&lng=en http://dx.doi.org/10.1590/S0103-21002013000400008.

12- Ursi ES, Gavão CM. Prevenção de lesões de pele no perioperatório: revisão integrativa da literatura. Rev Latino-Am Enfermagem [Internet]. 2006 [cited 2017 Jul 20]; 14(1):124-131. Available from: http://www.scielo.br/scielo.php?script=sci arttext\&pid=S0104-116920060001000178lng=en. http://dx.doi.org/10.1590/S010411692006000100017

13- Dutra RAA, Salomé GM, Alves JR, Pereira VOS, Miranda FD, Vallim VB et al. Using transparent polyurethane film and hydrocolloid dressings to prevent pressure ulcers. Journal ofWound Care. [Internet]. 2015 [cited 2017 Jul 20]:24(6):268-275. Available from: http://www.univas.edu.br/mpcas/egresso/publicacao/2016110711701118748690.pdf

14- Inoue KC. Matsuda LM. Custos de coberturas para a prevenção de úlcera por pressão sacral. Rev Bras Enferm [Internet]. 2016 [cited 2017 Jul 20]; 69(4):641-645. Available from: http://www.scielo.br/pdf/reben/v69n4/0034-7167-reben-69-04-0641. pdf

15- Hao DF, Feng G, Chu WL, Chen ZQ, Li SY. Evaluation of effectiveness of hydrocolloid dressing vs ceramide containing dressing against pressure ulcers. European Review for Medical and Pharmacological Sciences [Internet]. 2015 [cited 2017 Jul 20]; 19:936941. Available from: https://www.ncbi.nlm.nih.gov/pubmed/25855916

16- Linthwaite A, Bethell E. Managing pressure ulcers and moisture lesions with new hydrocolloid technology. British Journal of Nursing [Internet]. 2016 [cited $2017 \mathrm{Jul} 20$ ] 25(8):442-448. Available from: https://www.ncbi.nlm.nih.gov/pubmed/27126753

17- Inoue KC, Matsuda LM. Avaliação de custo-efetividade de dois tipos de curativos para prevenção de úlcera por pressão. Acta paul enferm [Internet]. 2015 [cited 2017 Jul 20]: 28(5):415-419. Available from: http://www.scielo.br/scielo.php?script=sci_ arttext\&pid=S0103-21002015000500415\&lng=en. http://dx.doi.org/10.1590/19820194201500070 